\title{
Correlation between the progression of optic disc and visual field changes in glaucoma
}

\author{
Stefano Miglior, Luca Brigatti, Cristina Lonati, Luca Rossetti, Chiara Pierrottet and Nicola Orzalesi \\ Department of Ophthalmology, University of Milan, Institute of Biomedical Sciences, S. Paolo Hospital, Milan, Italy
}

\begin{abstract}
Visual ficld test and optic disc evaluation are the standard examination techniques used to detect the onset and progression of glaucoma. This explorative study was performed to assess the temporal correlation between visual field and optic disc changes in eyes with ocular hypertension and well-established glaucoma. Eighty-six hypertensive and 16 glaucomatous eyes were followed up for a period of up to 9 years (average $4.4 \mathrm{yrs}$ ) using kinetic and computerized static perimetry and optic disc manual morphometry. Perimetric changes were based on a series of strict criteria and optic disc changes were based as a reduction in the baseline rim area/disc area ratio (R/D) measurement exceeding the $99 \%$ confidence interval for intraobserver reproducibility (7.7\%). Optic disc changes were found prior to visual field changes in four hypertensive eyes, whereas visual field changes were found prior to disc changes in six glaucomatous eyes $(\mathrm{p}=$ 0.042 ). The results of our explorative study suggest that quantitative optic disc analysis may be more sensitive than visual field examination in detecting early glaucomatous changes, whereas visual field examination may be more sensitive than quantitative optic disc analysis in detecting glaucomatous progressions in eyes with well established glaucoma. Curr. Eye Res. 15: 145-150, 1996.
\end{abstract}

Key words: follow-up; glaucoma; human; ocular hypertension; optic disc morphometry; visual field

\section{Introduction}

The onset and progression of glaucoma are generally assessed by means of visual field tests and optic disc evaluation. These procedures make it possible to identify the occurrence of a diffuse or localized depression of retinal sensitivity and of structural damage to the optic disc (enlargement of cupping and focal or diffuse narrowing of the neuroretinal tissue).

Correspondence: Dr. Stefano Miglior, Clinica Oculistica, Universita' degli Studi di Milano, Istituto di Scienze Biomediche, H. S. Paolo, Via di Rudini' 8, 20142 Milano, Iraly
Studies of human specimens showed that a certain number of retinal nerve fibers (RNF) are lost before the onset of detectable visual field defects (1). Sommer et al. (2) observed the presence of retinal nerve fiber layer defects six years before field loss in $60 \%$ of their patients with ocular hypertension at baseline. As a good clinical correlation between progressive RNF loss and progressive morphometric optic disc changes in glaucoma has been observed $(3,4)$, a decrease in rim disc area over time may be assumed as an estimate of progressive RNF atrophy. Odberg and Riise (5), in reporting the results of the first prospective study of sequential optic disc stereophotography, confirmed the impression that, in the earliest stages of the disease, a decrease in the neuroretinal rim area of the disc may be observed before the occurrence of visual field defects.

Only a few studies have quantitatively compared the rates of disc (6) and visual field changes among patients at high risk of developing glaucoma and patients with glaucoma $(7-9,10)$. Their results indicate that measurable optic disc changes precede visual field loss in the early glaucomatous stages. However, in more advanced stages, serial optic disc evaluation seems not as sensitive as visual field examination in detecting the further progression of glaucoma (10).

The aim of this explorative study was to quantify the rates of onset and progression of optic disc damage and visual field loss in two distinct populations of eyes: the first with ocular hypertension but a normal visual field and optic disc; the second with well-established glaucomatous visual field and optic disc changes.

\section{Subjects and methods}

A series of consecutive Caucasian subjects with a best corrected visual acuity $\geq 0.7$, a refraction range $-6 /+4$ diopters, no optic disc malformations (e.g., tilted disc), phakic eyes with clear optic media, no major ocular and systemic diseases, were enrolled from the Glaucoma Service of our Department. All of the subjects gave their informed consent to be entolled in the study, which was performed according to the principles of the Declaration of Helsinki regarding human subjects. All of the patients 
underwent a complete ophthalmological examination, which included keratometry (Javal-Schiotz) and axial length measurement (standard A-scan ultrasonography). In all cases, intraocular pressure (IOP), measured by means of Goldmann applanation tonometry, was calculated as the median of five measurements performed during diurnal monitoring (between 8 a.m. and 7 p.m.).

The visual field examinations were performed by means of Goldmann manual kinetic and computerized static perimetry (Perikon PCL 90, Optikon, Rome) (11, 12). The manual technique was used until the computerized perimeter became available in 1990, and was used to test four isopters (II-4, I-4, I-3, I-2). The patients tested with the Goldmann perimeter were followed throughout the study with the Goldmann perimeter, and the patients tested with static perimetry were followed entirely throughout the study with the static perimetry.

The computerized visual field examinations were performed using the 'DS 30-II' program of the Perikon PCL 90 system, which is based on the assessment of the full threshold in 76 points of the central $30^{\circ}$ area. The threshold value at every test point is measured using the standard double-threshold crossing (bracketing) method. Stimulus luminance is varied in a stepwise manner ( $4 \mathrm{~dB}$ steps) until there is a change in the patients response, when the procedure is continued in the opposite direction ( $2 \mathrm{~dB}$ steps) until there is a second change in response. The starting level for each measurement is determined by the adjacent tested-points. Every threshold value that differs by more than $4 \mathrm{~dB}$ from that expected according to the values of the neighboring points is thresholded again $(11,12)$.

The following parameters were used in all cases: stimulus size III (Goldmann target), stimulus duration $200 \mathrm{~ms}$, background luminance $31.5 \mathrm{asb}$. The visual field evaluations were performed by providing the patient with the current refractive correction and near add, and with a pupil dilation of $3 \mathrm{~mm}$ or more. All of the patients underwent a series of three field examinations (over a time period of no more than thirty days) in order to overcome problems related to the learning effect.' All of the eyes included in the study had false positive or false negative rates of less than $16 \%$.

Optic disc photography and morphometry were performed as previously described (13-15). For each optic disc, nonsimultaneous stereoscopic pairs of black \& white photographs were obtained by using a right-to-left lateral shift of $3 \mathrm{~mm}$ (corneainduced parallax method). The photographs were taken through a dilated pupil ( $\geq 5 \mathrm{~mm}$ ) by means of a Topcon TRC 50VT (Tokyo, Japan) fundus camera with a $35^{\circ}$ view angle. Previous comparison of simultaneous vs. non-simultaneous stereoscopic slides have shown a high correlation for rim area assessment $(r=0.87,95 \% \mathrm{CI}=0.78-0.92)(14)$. Contrast enhancement of the cup boundaries was obtained using the panchromatic Ilford PAN F (50 ASA) and a green illumination $(530 \mathrm{~nm})$ coupled with a barrier filter with $95 \%$ peak transmission between 540 and $625 \mathrm{~nm}$.

The boundaries of the optic disc, cup and neuroretinal rim were defined according to Britton et al. 16) and the results of the meeting on the 'Biomorphometry of the Optic Nerve' (17). The disc area was measured inside the inner circumference of the white scleral lip. The cup area was delimited by a contour line indicating the change of slope on the edge of the neuroretinal rim. If a vessel unattached to nerve fiber tissue was found within the cup, it was recorded as a part of the cup and not as a part of the rim (16).

The disc and cup boundaries were drawn on a transparent sheet lying on a slide projector (Slidex file projector, Slidex Corp. Inc., Tokyo) while simultaneously evaluating a couple of stereoscopic images of the same disc by means of a stereo viewer. The two outlined contours were then scanned and the enclosed area calculated by means of a computer equipped with a dedicated software written by one of the authors (L. B.). The actual size of the optic disc parameters was calculated using Littmans equations, which take into account ocular axial length and keratometry (18).

The neuroretinal rim area was calculated by subtracting the area of the cup from that of the disc, and the rim/disc area ratio (R/D) was also calculated. The definition of the cup contour in eyes without any evident change of slope was made easier by the photographic contrast enhancement and the stereoscopic evaluation of the photographs (13-15). When five pairs of randomly chosen photographs of the sample population were measured five times by the same observer, the coefficient of variation was 0.029 for R/D and 0.037 for rim area.

Ocular hypertension (OHT) at baseline was defined as an intraocular pressure of more than $21 \mathrm{~mm} \mathrm{Hg}$ without therapy and a normal visual field [i.e. the absence of any visual field defect at kinetic perimetry, or a Mean Defect (MD) $<4 \mathrm{~dB}$ and Corrected Loss Variance (CLV) $<6 \mathrm{~dB}^{2}$ at static perimetry].

Primary open angle glaucoma (POAG) at baseline was defined on the basis of well established, reproducible glaucomatous visual field defects. When the baseline examination was performed using the Goldmann perimeter, glaucoma was defined according to Epstein et al. (19): a nasal step $>8^{\circ}$; a paracentral scotoma $>5^{\circ}$ (in the largest diameter); a peripheral constriction of the outermost isopter $>10^{\circ}$ or a central constriction of the baseline isopter (used to define central $30^{\circ}$ ) of $\geq 10^{\circ}$. When the baseline examination was performed using the static perimeter, glaucoma was defined as the presence of a MD $>8 \mathrm{~dB}$ or CLV $>10 \mathrm{~dB}^{2}$.

In assessing disc change, the $\mathrm{R} / \mathrm{D}$ ratio was used as the reference parameter because of its lowest measurement variability and the fact that it has been shown to be unaffected by age (20). Progressive disc damage was defined as a reduction in the baseline R/D measurement exceeding the $99 \%$ confidence interval for intraobserver reproducibility $(7.7 \%)$.

In the OHT group, the onset of a visual field change at the Goldmann perimeter, was based on the occurrence of a nasal step $\left(\geq 5^{\circ}\right)$, or a paracentral scotoma $\left(\geq 5^{\circ}\right)$, or constriction of the peripheral or central isopter $\left(\geq 5^{\circ}\right)$, and had to be confirmed at the next examination.

In the POAG group, the progression of visual field changes, was based on a deepening of the nasal step $\left(\geq 5^{\circ}\right)$, an enlargement of the paracentral scotoma $\left(\geq 5^{\circ}\right)$, or further constriction of the peripheral or central isopter $\left(\geq 5^{\circ}\right)$, which also had to be confirmed at the next examination.

Static perimeter visual field changes were defined according to Zeyen and Caprioli (9) as a reproducible loss $\geq 10 \mathrm{~dB}$ in two 
or more contiguous points in Bjerrums area, or a reproducible loss $\geq 5 \mathrm{~dB}$ in three or more contiguous points in Bjerrums area, or a $10 \mathrm{~dB}$ difference across the nasal horizontal midline in two or more adjacent locations, that did not exist previously. The visual field change had to be confirmed at the next examination also in this case.

Patients underwent optic disc photography and visual field examinations, and were evaluated for the presence of lens opacities every year. Follow-up lasted for a minimum of two years. Visual acuity $\geq 0.7$ and clear optic media were required throughout the duration of the study.

All of the OHT and glaucomatous patients were under topical treatment with $\beta$-blockers alone or in association with adrenergic agonists. The average IOP of all the eyes ranged between 12 and $24 \mathrm{~mm} \mathrm{Hg}$ throughout the study.

Analysis of proportions ( $\mathrm{z}$ ) was carried out in order to compare intra- and inter-groups proportions. Fishers exact test was used when comparing data in $2 \times 2$ contingency tables. Differences between means were estimated by means of $95 \%$ confidence limits. The level of statistical significance for these calculations was $\mathrm{p}<0.05$.

\section{Results}

Eighty-two patients met all of the inclusion criteria, for a total of 116 eyes. The OHT group consisted of 70 patients ( 39 females and 31 males; age range 45-65 years) and 98 eyes; the POAG group of 12 patients ( 7 females and 5 males; age range 49-63 years) and 18 eyes. Ten patients ( 12 eyes) in the OHT and 2 ( 2 eyes) in the POAG group did not complete the required minimum follow-up mainly due to problems related to an insufficient quality of optic disc photography or unreliable visual field examinations (false positives or negatives $>16 \%$ ). The data from these patients were not considered in the analysis. The mean follow-up for both groups was 4.4 years (range 2-9 years).

Morphometric measurements of optic disc parameters showed that the two groups had significantly different baseline cup and rim areas and $R / D$ ratio, but their disc sizes were not significantly different (Table 1).

In the OHT group, progression occurred in four eyes as assessed by changes in $R / D$ and in one as assessed by $R / D$ plus visual field changes. In the POAG group, visual field changes revealed progression in six eyes, visual field plus $R / D$ changes in two, and $R / D$ changes alone in one (Table 2).

Table 1. Optic disc morphometry at baseline

\begin{tabular}{lccc}
\hline & OHT & POAG & p \\
\hline disc* & $2.69( \pm 0.65)$ & $2.64( \pm 0.71)$ & n.s. \\
cup* & $0.46( \pm 0.32)$ & $1.1( \pm 0.59)$ & $<0.01$ \\
rim* & $2.22( \pm 0.63)$ & $1.56( \pm 0.69)$ & $<0.01$ \\
R/D & $0.83( \pm 0.13)$ & $0.6( \pm 0.18)$ & $<0.01$ \\
\hline
\end{tabular}

OHT: ocular hypertension group; POAG: primary open angle glaucoma group. $* \mathrm{~mm}^{2}$.
Table 2. Occurrence of R/D and visual field changes (mean follow-up 4.4 years). 81 OHT and 7 POAG eyes did not show any change

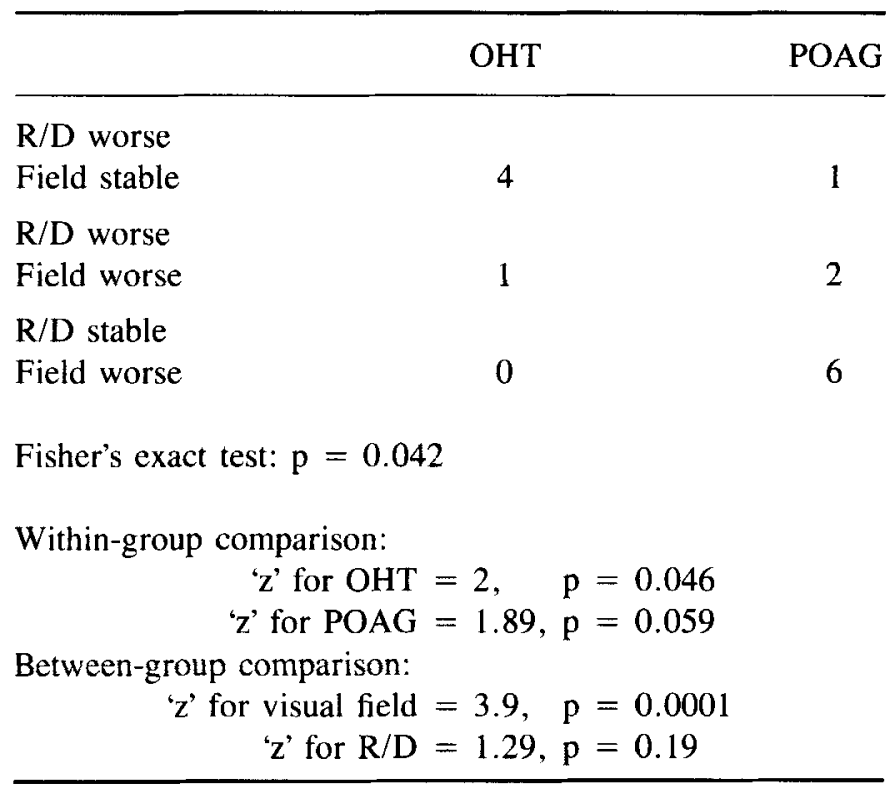

OHT: ocular hypertension group; POAG: primary open angle glaucoma group.

The two examination systems had different degrees of sensitivity according to the stage of the disease. In very early stages (among the OHT patients), optic disc morphometry was more sensitive than visual field examination $(\mathrm{p}=0.046)$. In glaucomatous eyes, visual field testing achieved the best score although this difference did not reach statistical significance $(p=0.059)$. The difference in the sensitivity of visual field investigations between the two groups was highly significant $(p=0.0001)$, whereas the difference in the sensitivity of optic disc morphometry was not. The correlation between the different examination techniques and the two different stages of the disease was statistically significant ( $p=0.042$ ), as measured by means of Fisher's exact test.

\section{Discussion}

It has been suggested that, when assessed using the available diagnostic procedures, the progression of the loss of retinal sensitivity and the decrease in the rim area of the optic disc varies according to the different stages of the disease $(9,10,21)$. In early stages, changes in the optic disc can be seen before the occurrence of perimetric abnormalities; in advanced stages, progressive loss of visual function may take place with no detectable change in the appearance of the optic disc.

The present study was performed to compare visual field examination and optic disc morphometry in the assessment of glaucoma onset and progression. Our results show the occurrence of measurable optic disc damage before the onset of visual field defects in hypertensive eyes, and progressive visual field loss before the development of measurable optic disc deterioration in eyes with well-established glaucoma. A concern of this 
study is the limited number of glaucomatous patients, and the limited number of 'converters' in both groups, which makes it difficult to draw conclusions, especially considering that primary open angle glaucoma is a relatively common disease. However, the outcome was statistically significant $(\mathrm{p}=0.042)$, with a power of the statistic test equal to $76 \%(1-\beta)$, despite the limited number of eyes with disc or field progression in the two groups. This suggests that the differences in detecting disc and field progressions (with the given criteria) in OHT and POAG eyes were substantially wide.

These findings confirm the observations of Zeyen and Caprioli (9), and Caprioli (10) and indirectly support those of Sommer et al. (2): in eyes at high risk for glaucoma, disc damage can be detected prior to the development of field loss, as evaluated by means of conventional perimetry. Conversely they disagree with the findings of Quigley et al. (4), but in their study optic disc assessment was performed qualitatively and with the evaluation of cup/disc ratios on colour photographs. Furthermore, our results are in agreement with the observations of Caprioli about the higher sensitivity of visual field testing in detecting disease progression among patients with well-established glaucoma (10).

This may be due to the fact that the amount of nerve fiber loss required to induce a decrease in retinal sensitivity may be different in the early and advanced stages of the disease. In the initial stages, it has been observed that a large number of nerve fibers have to be lost before visual field defects appear $(1,2)$, possibly as a result of the low sensitivity of the visual field examination itself during these stages or because of the overlapping of retinal receptive fields. The stimulation of individual receptors may affect the firing rate of a number of ganglion cells (2224 ), thus allowing the visual signal to be carried by different nerve fibers from overlapping fields and, in this case, the atrophy of a certain number of ganglion fibers may be compensated for by the contiguous fibers (at least partially).

Conversely, in more advanced stages characterized by wellestablished disc and visual field deterioration, the further loss of a very small number of nerve fibers may have dramatic effects in terms of visual field changes. In this case, the loss of just a few residual ganglion cells (possibly undetectable at the level of the neuro-retinal rim tissue of the disc) may eventually lead to the definite loss of large receptive fields.

However, our results may have been affected by differences in the sensitivity of our definitions of visual field and optic disc changes in both the early and advanced stages of glaucoma.

The kinetic perimetry criteria used to define glaucomatous visual field changes, which refer to the very strict indications of Epstein et al. (19), may not be as sensitive as those used in computerized systems; the occurrence or deepening of small localized scotomas may be missed by the first and detected by the second. Moreover, even when computerized perimetry is used, the resolution and current strategies of central area full threshold examinations may be less accurate than expected (25). A strategy aimed at exploring Bjerrumis area with a higher resolution of raster points might increase the sensitivity of visual field assessment in the early stages of the disease, as localized RNFL defects probably appear in these regions ( $48 \%$ of all of the incident cases of RNFL defects according to Tuulonen et al. [3]).
On the other hand, optic disc morphometry is highly sensitive in detecting changes when there is a lot of rim tissue, but appears to be inefficient when much of the rim tissue is lost and only a very small amount of neural tissue loss needs to be detected in order to document the progression of disc changes. In this case, only a lower coefficient of variation of the rim area or $R / D$ measurements would allow the statistically significant detection of smaller losses of the rim area, thus increasing the sensitivity of optic disc morphometry in the advanced stages of glaucoma. Preliminary results from our laboratory have shown that the coefficient of variation of $R / D$ ratio, measured on disc images acquired using a Scanning Laser Ophthalmoscope, may be about 0.005 , which is consistent with an upper $99 \% \mathrm{CL}$ of about $0.01-0.02(26)$.

The early detection of visual field and optic disc deterioration and progression is crucial to the management of high-risk and glaucomatous patients. The standard diagnostic procedures are mainly based on the computerized visual field assessments (which allow the evaluation of retinal sensitivity of a well defined retinal area), and the qualitative evaluation of various optic disc parameters (27). The introduction of manual optic disc morphometry may add new quantitative information concerning optic discs (28-31, 13-16) and allows them to be more precisely monitored over time $(3,6,9,10,30)$.

With the given criteria of optic disc changes and visual field loss, our results suggest that measurable optic disc changes may be detected earlier than visual field loss in ocular hypertensive eyes, whereas progressive visual field changes may be detected earlier than measurable optic disc changes in cases of wellestablished glaucoma. However, as our study did not address the temporal sequence of events, it is not possible to add any definitive conclusions about the adoption of specific and possibly expensive "optic disc evaluation" protocols which may reveal changes at an earlier date, but perhaps only 6 or 12 months earlier than the visual field. Thus, it may be easier and more economical to utilize the visual field protocol and accept this hypothetical slight difference in the time sequence.

\section{References}

1. Quigley, H. A., Addicks, E. M. and Green, V. R. (1982) Optic nerve damage in human glaucoma. III. Quantitative correlation of nerve fiber loss and visual field defects in glaucoma, ischemic neuropathy, papilledema, and toxic neuropathy. Arch. Ophthalmol. 100, 135-146.

2. Sommer, A., Katz, J., Quigley, H. A., Miller, N. R., Robin, A. L., Richter, R. C. and Witt, K. A. (1991) Clinically detectable nerve fiber atrophy precedes the onset of glaucomatous field loss. Arch. Ophthalmol. 109, 77-83.

3. Tuulonen, A., Airaksinen, P. J. (1991) Initial glaucomatous optic disc and retinal nerve fiber layer abnormalities and their progression. Am. J. Ophthalmol. 111, 485-490.

4. Quigley, H. A., Katz, J., Derick, R. J., Gilbert, D. and Sommer, A. (1992) An evaluation of optic disc and nerve fiber layer examinations in monitoring progression of early glaucoma damage. Ophthalmology, 99, 19-28.

5. Odberg, T. and Riise, D. (1985) Early diagnosis of glaucoma: 
the value of successive stereophotography of the optic disc. Acta Ophthalmol. 63, 257-268.

6. Airaksinen, P. J., Tuulonen, A. and Alanko, H. I. (1992) Rate and pattern of neuroretinal rim area disease in ocular hypertension and glaucoma. Arch. Ophthalmol. 110, 206210.

7. Harbin, T. S. Jr., Podos, S. M., Kolker, A. E. and Becker, B. (1976) Visual field progression in open angle glaucoma patients presenting with monocular field loss. Trans. Am. Acad. Ophthalmol. Otolaryngol. 81, 253-257.

8. Kass, M. A., Kolker, A. E. and Becker, B. (1976) Prognostic factors in glaucomatous visual field loss. Arch. Ophthalmol. 94, 1274-1276.

9. Zeyen, T. G. and Caprioli, J. (1993) Progression of disc and field damage in early glaucoma. Arch. Ophthalmol. 111, 62-65.

10. Caprioli, J. (1994) Clinical evaluation of the optic nerve in glaucoma. Tr. Am. Ophth. Soc. 92, 589-641.

11. Zingirian, M., Gandolfo, E., Capris, P. and Mattioli, R. (1991) Perikon PCL 90: a new automatic perimeter. In Perimetry Update, 1990-1991 (Eds. Mill, R., Heijl, A.). Pp. 403-408. Kugler Publisher, Amsterdam, New York.

12. Zingirian, M., Gandolfo, E., Capris, P. and Mattioli, R. (1991) Computerized system for static and kinetic automatic perimetry. Europ. J. Ophthalmol. 1, 181-186.

13. Brigatti, L., Bottoni, F., Miglior, S. and Orzalesi, N. (1991) Technical procedures and software for magnificationcorrected morphometry of optic disc photography. Ophthalmologica, 202, 33-37.

14. Orzalesi, N., Miglior, S., Brigatti, L. and Bottoni, F. (1991) Manual morphometry in normal and glaucomatous eyes. In Glaucoma-Concepts in Evolution (Eds. Bonomi, L. and Orzalesi, N.). Pp. 51-69. Kugler Publisher, Amsterdam, New York.

15. Miglior, S., Brigatti, L., Velati, P., Balestreri, C., Rossetti, L., Bujtar, E. and Orzalesi, N. (1994) Relationship between morphometric optic disc parameters, sex and axial length. Curr. Eye Res. 13, 119-124.

16. Britton, R. J., Drance, S. M., Schulzer, M., Douglas, G. R. and Mawson, D. K. (1987) The area of the neuroretinal rim of the optic nerve in normal eyes. Am. J. Ophthalmol. 103, 497-504.

17. Jonas, J. B., Airaksinen, J. P. and Robert, Y. (1988) Definitionsentwurf der intra- und parapapillaren parameter für die biomorphometrie des nervus opticus. Klin. Monatsbl. Augenheilkd. 192, 621.

18. Littmann, H. (1988) Zur bestimmung der wahren große eines objektes auf dem hintergrund des lebenden auges. Klin. Monatsbl. Augenheilk. 192, 66-67.

19. Epstein, D. L., Krug, J. H., Hertzmark, E., Remis, L. L. and Edelstein, D. J.: A long-term clinical trial of timolol therapy versus no treatment in the management of glaucoma suspects. Ophthalmology, 96, 1460-1467.

20. Tsai, C. S., Ritch, R., Shin, D. H., Wan, J. Y. and Chi, T. (1992) Age related decline of disc rim area in visually normal subjects. Ophthalmology, 99, 29-35.

21. Caprioli, J. (1991) Quantification of optic nerve damage in glaucoma. In Glaucoma-Concepts in Evolution (Eds. Bonomi, L. and Orzalesi, N.). Pp. 77-90, Kugler Publisher, Amsterdam, New York.

22. Ikeda, H. and Hill, R. M. (1971) Can a peripheral ganglion cell respond differentially to images in and out of focus? Nature, 229, 557-558.

23. Ikeda, H. and Wright, M. J. (1971) How large is the receptive field of a single retinal ganglion cell? J. Physiol. 217, 52-53.

24. Ikeda, H. and Wright, M. J. (1972) Differential effects of refractive errors and receptive field organization of central and peripheral ganglion cells. Vision. Res. 12, 1465-1475.

25. Tuulonen, A., Lehtola. J. and Airaksinen, P. J. (1993) Nerve fiber layer defects with normal visual fields. Do normal optic disc and normal visual field indicate absence of glaucomatous abnormality? Ophthalmology, 100, 587-598.

26. Miglior, S., Rossetti, L., Lonati, C. and Orzalesi, N. (1995) Reproducibility of optic disc morphometry by scanning laser ophthalmoscopy. (Abstract). Invest. Ophthalmol. Vis. Sci. 36 (Suppl.), 970

27. Spaeth, G. L. (1993) Development of glaucomatous changes of the optic nerve. In The Optic Nerve in Glaucoma (Eds. Varma, R. and Spaeth, G. L.). Pp. 63-81. J.B. Lippincott Company Publisher, Philadelphia.

28. Airaksinen, P. J., Drance, S. M. and Schulzer, M. (1985) Neuroretinal rim area in early glaucoma. Am.J. Ophthalmol. $99,1-4$.

29. Jonas, J. B., Gusek, G. C. and Naumann, G. O. H. (1988) Optic disc, cup and neuroretinal rim size, configuration and correlation in normal eyes. Invest. Ophthalmol. Vis. Sci. 29, $1151-1158$.

30. O'Connor, D. J., Zeyen, T. and Caprioli, J. (1993) Comparison of methods to detect glaucomatous optic nerve damage. Ophthalmology, 100, 1498-1503.

31. Tuulonen, A., Takamoto, T., Wu, D. and Schwartz, B. (1987) Optic disc cupping and pallor measurements of patients with a disk hemorrhage: a controlled follow-up study. Am. J. Ophthalmol. 103, 505-511. 